\title{
Comparative Assessment of Virtual Track Circuit Based on Image Processing
}

\author{
Florin Codruţ Nemţanu ${ }^{1} \cdot$ Dorin Laurenţiu Bureţea ${ }^{1} \cdot$ Luigi Gabriel Obreja $^{1}$
}

Received: 9 March 2015/Revised: 17 May 2015/Accepted: 26 May 2015/Published online: 15 August 2015

(C) The Author(s) 2015. This article is published with open access at Springerlink.com

\begin{abstract}
For urban rail track, it is important to detect the presence of the tram or light train in black spots (like urban tunnels, bridges and low visual contact). The classical solution is to use track circuit which is safety oriented designed. The paper proposes a virtual track circuit as an alternative solution. For this proposal a comparative assessment was done to identify the main issues of this solution. For both systems analysed the authors defined and calculated two special functions: one is safety function which is a probability function (together with a distribution function) and the second one is error function which has the same type as previous one.
\end{abstract}

Keywords Virtual track circuit · Probability function · Comparative assessment $\cdot$ Railway safety

\section{Introduction}

One of the most important systems in our society is transport system. This system is a very complex one and it is composed by different transport modes. In this paper one important problem is addressed and this problem is related with urban transport and railway transport for urban area. Safety in transport systems is the main issue which is addressed by different systems and the main objective of those systems is to permit the movement of persons and

Florin Codruț Nemțanu

florin.nemtanu@ieee.org

1 Transport Faculty, University Politehnica of Bucharest, Bucharest, Romania

Editor: Baoming Han goods without any danger or threat [1]. A system which is able to increase the level of safety in railway transport system (for urban area in this case) is the track circuit which is a sensor installed to collect information about the presence of the rolling stock on a given controlled area [2].

The image processing and communication systems are now very well developed and designed and they are able to support applications for safety and railway safety (the majority of applications are developed for road transport) [3]. Pattern recognition, in terms of image analysis, could be a good solution to detect the presence of the rolling stock on a specific monitored area. The application of pattern recognition could increase the efficiency of the method in terms of providing additional information about an object placed on the rails, the physical obstacle between the camera and the rails. Various methods of pattern recognition were defined and tested and one of them could be selected to be applied in this research (the next stage of the research) [4]. This is the reason to introduce a new type of track circuit which is based on image processing. The main issue of this virtual track circuit is to demonstrate that this solution is able to provide the same level of safety as classical track circuit based on using tracks as wires for an emitter-receiver system. This demonstration is based on comparative assessment of those two different sensing solutions (a model of this assessment came from decision support systems domain) [5].

The authors defined the two main hypotheses of this research as following:

- The virtual track circuit is a safety oriented system and it is able to provide the same level of safety where they installed the system.

- The probability functions and a comparative assessment could provide enough information to support the first hypothesis. 
The method of comparative assessment is based on, firstly, establishing a reference, in the case of this paper the reference is the track circuit and its specific probability functions, and secondly to compare two probability functions associated with those two different types of track circuit.

In Fig. 1 a principle scheme of track circuit is presented with the main components, these components will be defined by their variable. The principle of this track circuit is: the tram which is ocupping the track will have a similar effect as an electrical shunt and the signal emitted by $\mathrm{E}$ is no longer received by $\mathrm{R}$, which means Red light is turning on and the next tram is stopped.

An equivalent model (Fig. 2) of this track circuit is proposed by authors in terms of defining probability function. The components of this model are: emitter (E), connectors (Con), tracks as wires (Track), receiver (R) and control subsystem (Ctrl).

The second track circuit, the virtual one, is described in Fig. 3 and the principle is: the tram is detected on specific area by a camera which is able to process the information and to send the information to a control subsystem and the red light is turned on.

An equivalent model is proposed and the structure (presented in Fig. 4) has the following components: Track

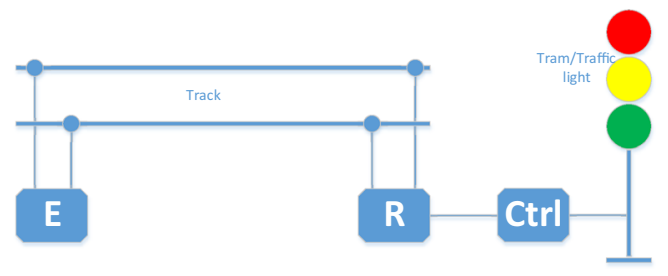

Fig. 1 The principle of track circuit

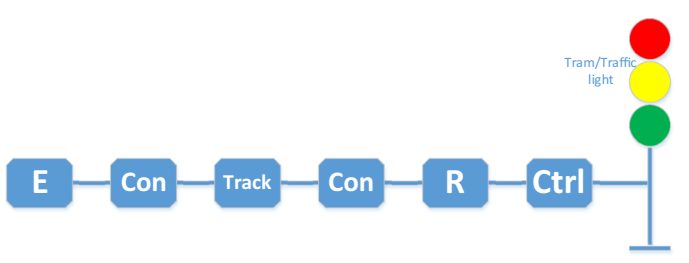

Fig. 2 Equivalent model of track circuit

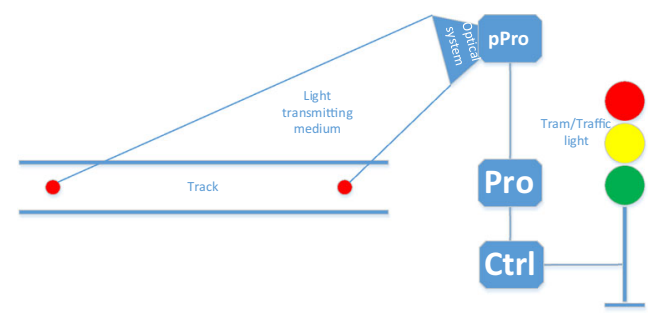

Fig. 3 The principle of virtual track circuit

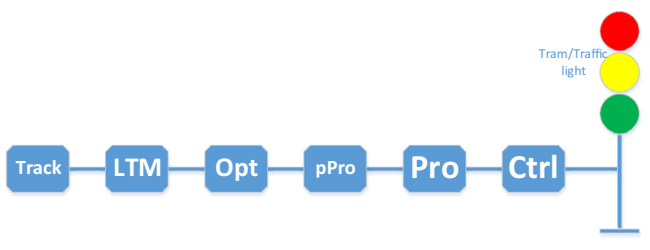

Fig. 4 Equivalent model of virtual track circuit

(Track), light transmission medium (LTM), optical system (Opt), pre-processing (pPro), image processing (Pro) and control subsystem (Ctrl).

\section{The Mathematical Instrument for Comparative Assessment}

The probability is defined in terms of likelihood of a specific event. If $X$ denotes an event, the probability of occurrence of the event $X$ is denoted by $P(X)$ [6].

$P(X)=\lim _{n \rightarrow \infty} \frac{m}{n}$

where $m$ is the number of the successful occurrences and $n$ is the number of observations.

$0 \leq P(X) \leq 1$

A probability density function is a function $f(x)$ defined on interval $(a, b)$ and having the following properties [7, 8]:

$f(x) \geq 0$ for every value of $x$

$\int_{a}^{b} f(x)=1$

A continuous random variable $X$ admits a probability function $f$ if for every $c$ and $d$,

$P(c \leq X \leq d)=\int_{c}^{d} f(x) d x$

Let $X$ be a $K \times 1$ continuous random vector. The joint probability density function of $X$ is a function $f_{X}: R^{K} \rightarrow$ $[0, \infty)$ such that:

$$
\begin{aligned}
P & \left(X \in\left[a_{1}, b_{1}\right] \times \cdots \times\left[a_{k}, b_{k}\right]\right) \\
& =\int_{a_{1}}^{b_{1}} \cdots \int_{a_{k}}^{b_{k}} f_{X}\left(x_{1}, \ldots, x_{k}\right) d x_{k} \ldots d x_{1}
\end{aligned}
$$

A discrete probability distribution shall be understood as a probability distribution characterised by a probability mass function [9]. The distribution of a random variable $X$ is discrete, and $X$ is then called a discrete random variable if:

$\sum_{a} P(X=a)=1$

where $a$ runs through the range of values of variable $X$. 
The probability that a discrete random variable $X$ takes on a particular value $x(P(X=x))$, is denoted $f(x)$. The function is called probability mass function. The probability mass function, $P(X=x)=f(x)$ of a discrete random variable $X$ is a function that satisfies the following properties: [10]

$$
\begin{aligned}
& P(X=x)=f(x)>0 \text { if } x \in \text { the support } S \\
& \sum_{x \in S} f(x)=1 \\
& P(X \in A)=\sum_{x \in A} f(x)
\end{aligned}
$$

\section{Comparative Assessment Based on Probability Functions}

The first step in this assessment is to identify the variable which are suitable to be part of this multivariate analysis of probability functions. In the Fig. 2 the authors presented a model with six components and a vector of six variables was defined based on Eq. (6).

$X=\left[x_{1}, x_{2}, x_{3}, x_{4}, x_{5}, x_{6}\right]$
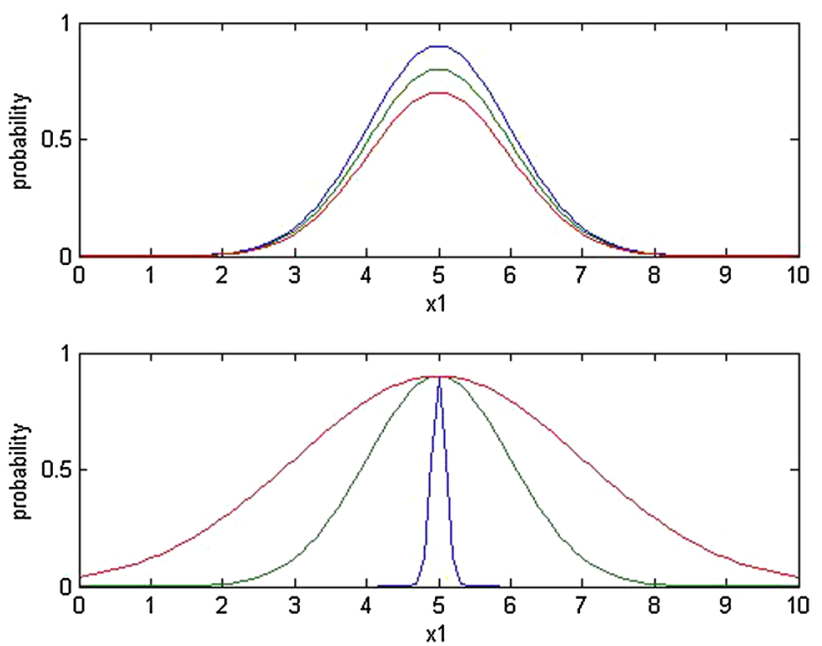

Fig. 5 Gaussian distribution of probability function - variable $x_{I}$
The definitions of this variables are available in the table as well as some other characteristics useful for the objectives of the research.

We assumed that the probability functions of all these variables have a Gaussian distribution (initially, after some iteration we can reconsider this distribution) and these functions have the graph represented in Fig. 5 and the graph is directly influenced by $\sigma$ (if sigma is near zero the probability of that event is near 1) and the probability associated with the variable in discussion.

The safety function is defined as the probability function calculated for entire chain of components (defined in equivalent model), that means for all specific variables (defined in Table 1) associated with these components, and this is a joint probability density function and, based on Eq. (6) and Table 1 the expression is:

$$
\begin{aligned}
P(X & \left.\in\left[a_{1}, b_{1}\right] \times \cdots \times\left[a_{7}, b_{7}\right]\right) \\
& =\int_{a_{1}}^{b_{1}} \cdots \int_{a_{7}}^{b_{7}} f_{X}\left(x_{1}, \ldots, x_{7}\right) d x_{1} \ldots d x_{7}
\end{aligned}
$$

The authors proposed a reference safety function (which is a probability function of a system to attempt a given level of safety) as a sum of the probability functions of all components of equivalent model. This joint probability density function will be considered as a reference for future comparative assessment of different systems. The safety function (in this model) has 6 components one for every components of the equivalent model and for other application the equivalent model could have more components (in fact, for real systems this number of components is the total number of devices, equipment and any other hardware or software entities). Using Matlab, the authors proposed a graphical representation of this safety function and a procedure to compare this figure with the figure of any other system (this figure is named foot print of safety function) [11]. The foot print of existing, accepted track circuit is the reference and the safety function of any other solution will be compared with this and the characteristics, in terms of safety, will be improved based on this comparative assessment. A partial safety function is the probability function for one component which is part of the safety
Table 1 Definition of variables $x_{i}$

\begin{tabular}{lllll}
\hline Variable & Definition & Component & Range & Probability \\
\hline$x_{1}$ & Power of emitted signal & Emitter & $0.5-1.5 \mathrm{~W}$ & Gaussian distribution \\
$x_{2}$ & Resistance of connector & Connection & $0-10 \Omega$ & Gaussian distribution \\
$x_{3}$ & Resistance of the ballast & Track & $0.8-2 \Omega / \mathrm{Km}$ & Gaussian distribution \\
$x_{4}$ & Resistance of connector & Connection & $0-10 \Omega$ & Gaussian distribution \\
$x_{5}$ & Amplification & Receiver & $0.1-1.5$ & Gaussian distribution \\
$x_{6}$ & Command signal (voltage) & Control & $10-14 \mathrm{~V}$ & Gaussian distribution \\
\hline
\end{tabular}



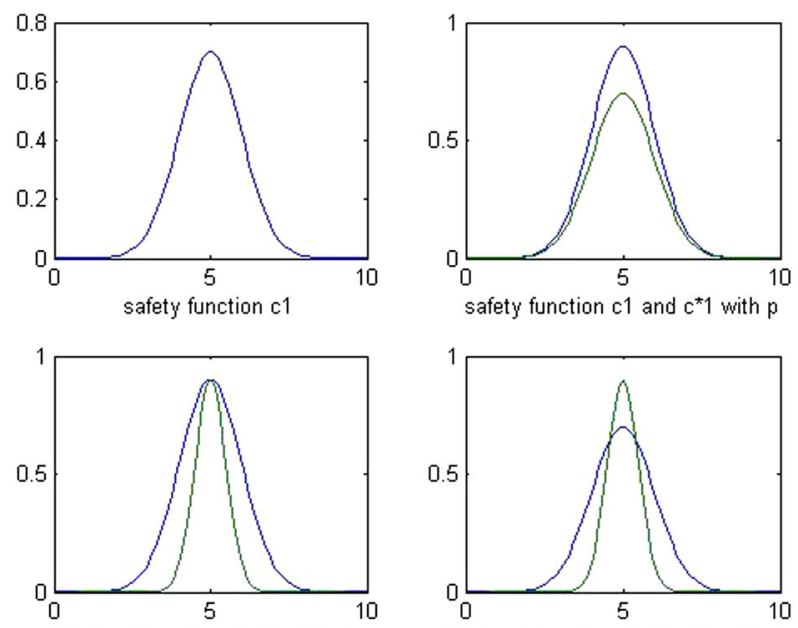

safety function $c 1$ and $c^{* 1}$ with sigma

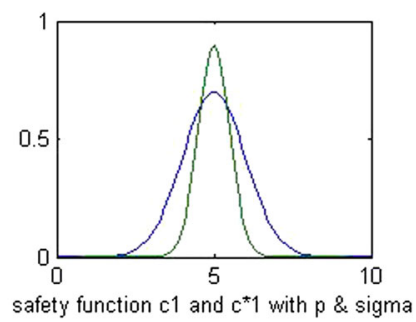

Fig. 6 Partial safety functions for one component $\left(c_{1}\right.$ and $\left.c_{1}^{*}\right)$

function. In this case $c_{1}$ is the component of track circuit used as reference and $c_{1}^{*}$ is the safety function for a similar component from the virtual track circuit (Fig. 6).

\section{Results}

The authors assumed that all variables have a Gaussian distribution of their probability functions or partial safety functions.

In the Fig. 4 the authors presented a model with six components for the virtual track circuit and a vector of six variables was defined based on Eq. (6).

$Y=[y 1, y 2, y 3, y 4, y 5, y 6]$

The definitions of this variables are available in the table as well as some other characteristics of the components of the equivalent model for a virtual track circuit (see Fig. 4).

The authors assumed that the weight of every partial safety function in entire safety function is equal. Based on this assumption all six components of the model of virtual track circuit (see Table 2) could replace all six components of the reference model (track circuit).

In Figs. 7 and 8 the partial safety functions of the track circuit defined as reference is revealed to provide an instrument to determinate the comparative safety function of virtual track circuit. To improve the safety of the track circuit the designer has to propose a component with a better partial safety function that means the shape of partial safety function has to be narrow (with a smaller sigma, in the case of Gaussian distribution of probabilities) and tall (higher probability for the normal state of the component) (Fig. 9).

A partial code in Matlab is presented by authors to show the software tool which is needed to generate safety image of the track circuit:

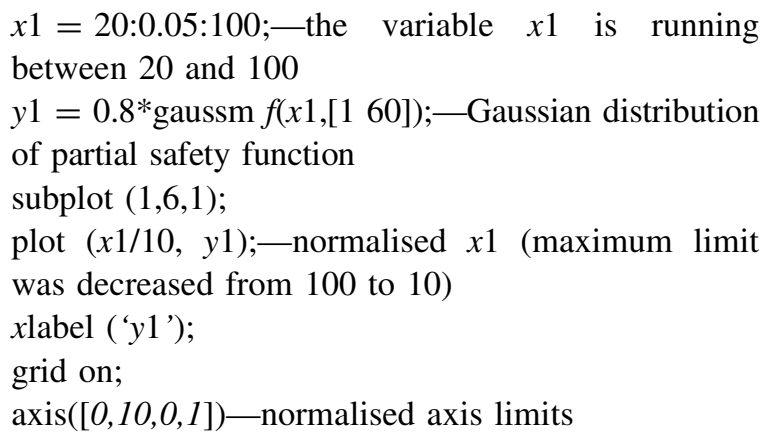

If the partial safety functions for component $c_{1}$ are compared, that means $x_{1}$ as reference and $y_{1}$ which comes from virtual track circuit, the virtual track circuit has a better partial safety function $y_{1}$, the shape is narrow and the probability is $0.8\left(0.1\right.$ more than $\left.x_{1}\right)$.

\section{Extension of the Solution}

In this paper, the authors presented two probability functions generated by a single variable per component of the equivalent model (for each type of track circuit). The authors proposed an extension of this solution through the extension of the number of variables per components of equivalent model. The extended number of variable will generate a modification of Eq. (6) as following:

- A set of vectors for the track circuit on per component (vectors of variables);

- composite variable could be defined to simplify the analysis;

Table 2 Definition of variables $y_{i}$

\begin{tabular}{lllll}
\hline Variable & Definition & Component & Range & Probability \\
\hline$x_{1}$ & Physical reference points and areas & Track & $20-100 \mathrm{~m}$ & Gaussian distribution \\
$x_{2}$ & The quality of light transmission medium—-the visibility & Light transmission medium & $10-100 \mathrm{~m}$ & Gaussian distribution \\
$x_{3}$ & Opacity of optical system & Optical system & $0-30 \%$ & Gaussian distribution \\
$x_{4}$ & Time of pre-processing & Pre-processing & $0.1-3 \mathrm{~s}$ & Gaussian distribution \\
$x_{5}$ & Time of image processing & Image processing & $0.1-5 \mathrm{~s}$ & Gaussian distribution \\
$x_{6}$ & Command signal (voltage) & Control & $10-14 \mathrm{~V}$ & Gaussian distribution \\
\hline
\end{tabular}



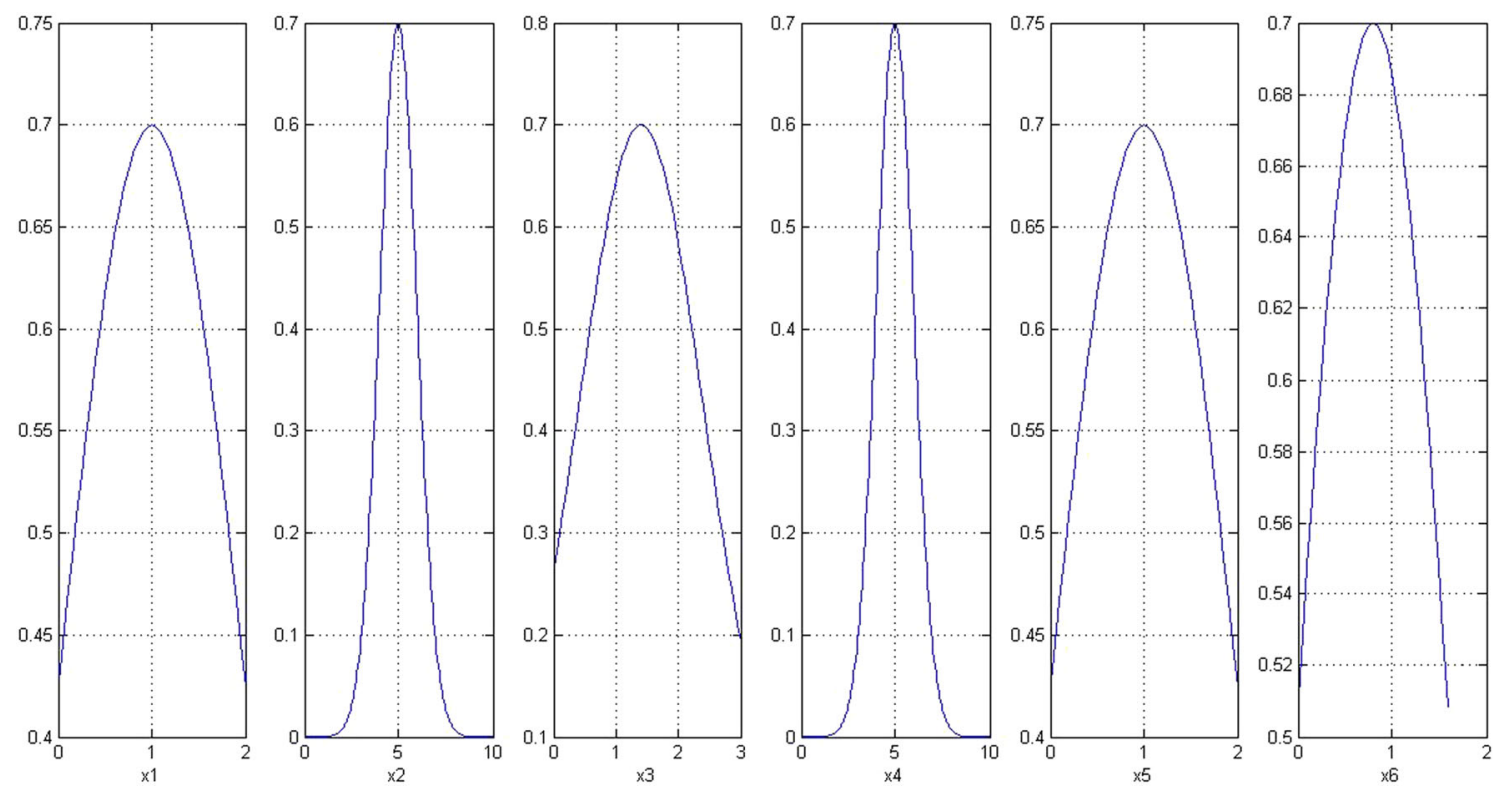

Fig. 7 Partial safety functions as components of safety function
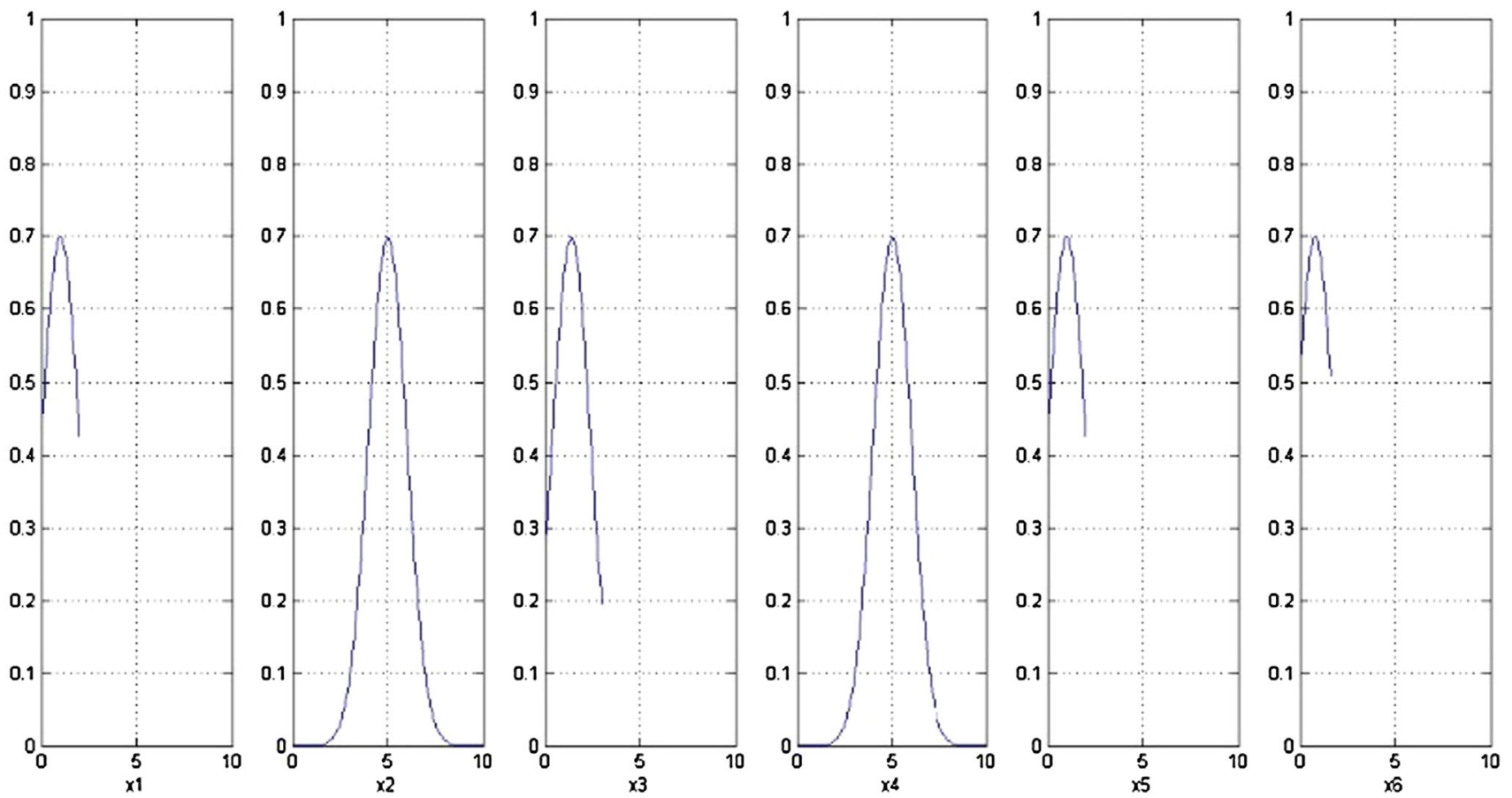

Fig. 8 Partial safety functions-safety image of the reference track circuit

- if the variables are discrete the integral becomes a sum.

- 3D representation (maximum 3 variables per components) is recommended.

In the case of two variable per component of equivalent model the following graph is generated as a representation of probability function as a part of safety function (Fig. 10).
Based on the functional models developed in the laboratory the comparative assessment will be done close to real conditions and the Gaussian distributions assumed in this paper will be replaced with the real distribution of the safety function (probability function). The same problem could be rose for another important analysis which is closed to safety, error function. 

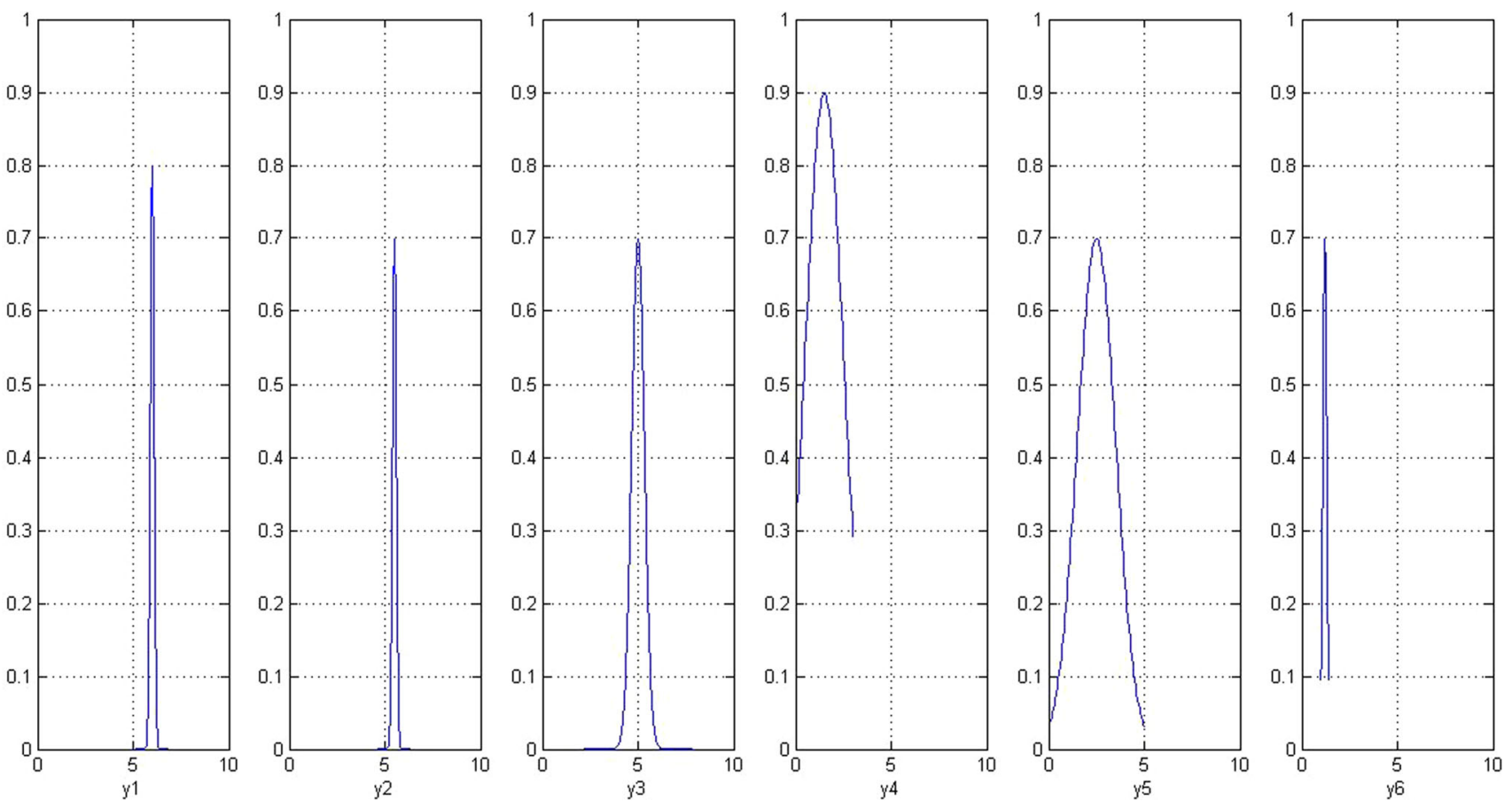

Fig. 9 Partial safety functions - safety image of the virtual track circuit

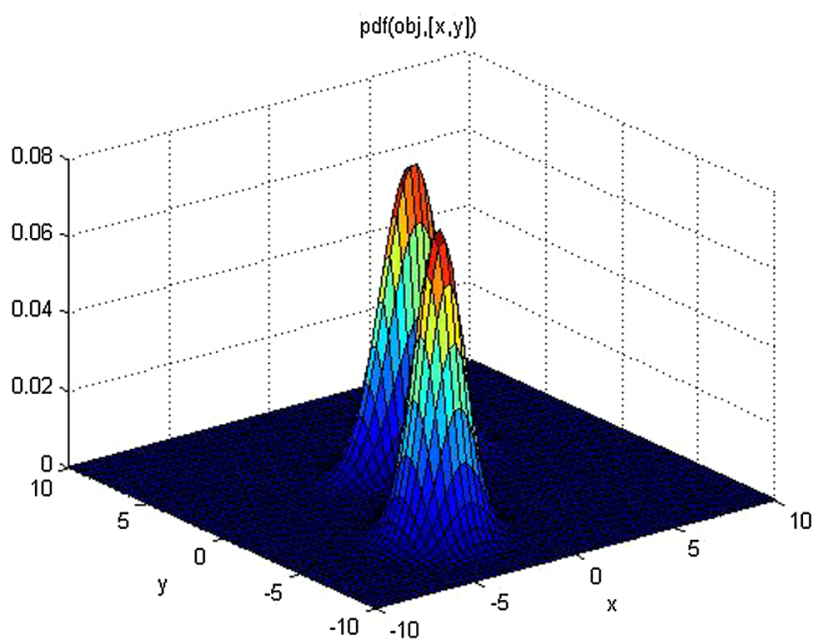

Fig. 10 Partial safety function for one component with two variables

\section{Conclusions}

The main idea of the paper is to elaborate a mathematical tool for comparative assessment of different technical systems based on probability theory, especially using probability functions.

The main advantage of this comparative assessment is the possibility to identify the weaknesses of the track circuit, in terms of partial safety functions, and to improve these components to obtain a better partial safety function (based on probability and sigma, in case of Gaussian distribution).

The reference will be considered an existing installed track circuit and a new track circuit, in this case a virtual one based on image processing will be compared to find the safety function and to improve partial safety functions. The research will be conducted to demonstrate the equivalence in terms of safety and reliability of virtual track circuit and the first propose is to use this virtual track circuit in urban tram network especially in branches of this network characterised by blind spot (tunnels, bridges etc.). The approach has to steps: the first one is to develop a test platform in laboratory-this platform will be based on an existing track circuit with a known safety function and a virtual lab based circuit with a calculated safety function - and the second one is to move this platform in real condition (the method will be tested in Bucharest, Romania in a tunnel of tram network).

The author presented in this paper the method for comparative assessment and the next step is to develop a functional model in laboratory for both, reference track circuit and the virtual track circuit.

The best safety function is defined also from the context perspective, which means urban railway has another context than interurban railway (in terms of speed and masses).

In these models all partial safety functions have a Gaussian distribution and for real equipment the real distribution has to be considered. 
Open Access This article is distributed under the terms of the Creative Commons Attribution 4.0 International License (http://crea tivecommons.org/licenses/by/4.0/), which permits unrestricted use, distribution, and reproduction in any medium, provided you give appropriate credit to the original author(s) and the source, provide a link to the Creative Commons license, and indicate if changes were made.

\section{References}

1. Flammini F (2012) Railway safety, reliability, and security: technologies and systems engineering. IGI Global

2. Franklin F, Nemtanu F, Teixeira PF (2013) Rail infrastructure, ITS and access charges. Res Transp Econ 41(1):31-42

3. Nagamalai D, Renault E, Dhanuskodi M (2011) Advances in digital image processing and information technology. In: first international conference on digital image processing and pattern recognition, DPPR 2011, Tirunelveli
4. Bezdek J, Keller J, Krisnapuram R, Pal N (2005) Fuzzy models and algorithms for pattern recognition and image processing. Springer, New York

5. Kiang MY (2003) A comparative assessment of classification methods. Decis Support Syst 35(4):441-454

6. Rao S (1996) Engineering optimization: theory and practice. Wiley, New York

7. www.zweigmedia.com (2012) [Online]. Available via http:// www.zweigmedia.com/RealWorld/index.html. Accessed 9 Oct 2014

8. Taboga M (2014) Statlect: the digital textbook. [Online]. Available via http://www.statlect.com/. Accessed 9 Oct 2014

9. Silverman B (1986) Density estimation for statistics and data analysis. CRC Press, London

10. STAT 414 (2014) The Pennsylvania State University. [Online]. Available via https://onlinecourses.science.psu.edu/stat414/node/ 57. Accessed 9 Oct 2014

11. MathWorks Documentation (2014) MathWorks, [Online]. Available via http://www.mathworks.com/help/index.html. Accessed 10 Oct 2014 